\title{
Nanoscale Devices and Systems
}

National Cancer Institute

\section{Source}

National Cancer Institute. Nanoscale Devices and Systems. NCI Thesaurus. Code C53672.

$R \& D$ that applies the principles of nanoscale science and engineering to create novel, or to improve existing, devices and systems. This PCA includes the incorporation of nanoscale or nanostructured materials to achieve improved performance or new functionality. To meet this definition, the enabling science and technology must be at the nanoscale, but the systems and devices themselves are not restricted to that size. 